\title{
Éthique enseignante et confiance
}

\section{HRONIQUE • Éthique en éducation}

Dans les textes légaux concernant le droit et l'éthique des enseignants, la question de la confiance est centrale. Il semble que les enseignants doivent agir en toutes situations pour conserver la confiance des élèves, de leurs parents, des membres de leur direction scolaire et du public en général. Les juges utilisent régulièrement l'argument de la perte de confiance pour justifier une sanction contre un enseignant. Par exemple, dans une cause récente, un juge souligne que la confiance des parents serait grandement ébranlée s'ils devaient apprendre que la commission scolaire a embauché un enseignant qui a été condamné pour trafic de stupéfiants, alors que, soutient-il, la consommation d'alcool et de stupéfiants est un problème social de première importance et un facteur de décrochage scolaire. Dans une autre cause, le juge déclare qu'une enseignante ne pourra réintégrer ses fonctions, puisque le lien de confiance avec son employeur a été entièrement brisé à la suite des vols qu'elle aurait commis.

Dans une célèbre cause qui date de 1996, Yves Audet, un enseignant d'éducation physique du niveau secondaire a été accusé d'avoir touché à des fins d'ordre sexuel, en contravention de l'alinéa 153(1)a) du Code criminel, une adolescente qui avait l'âge du consentement. Dans cette cause, les juges devaient déterminer si l'enseignant Audet était en position d'autorité et de confiance lorsqu'il a couché avec une élève à qui il avait enseigné durant les vacances estivales. Les juges de la Cour suprême du Canada ont considéré que l'enseignant était en position d'autorité et de confiance sur la jeune élève du fait qu'il allait lui enseigner de nouveau. Selon les juges du plus haut tribunal du pays, " [...] il n'existe aucune circonstance pertinente à la qualification de la nature de la relation entre 
l'enseignant et l'adolescente qui soit susceptible de soulever un doute raisonnable dans l'esprit du juge des faits quant à la situation de confiance dans laquelle l'enseignant se trouvait vis-à-vis de l'adolescente. Même si l'incident a eu lieu lors des vacances d'été, ces vacances venaient de débuter et les circonstances indiquent que l'enseignant allait de nouveau enseigner à l'adolescente. Il était donc tout au moins en situation de confiance vis-à-vis de celle-ci. Notre Cour est donc justifiée d'exercer le pouvoir que lui confère le par.686(4) du Code criminel de casser le verdict d'acquittement prononcé par le juge du procès et d'y substituer un verdict de culpabilité puisque n'eût été l'erreur de droit commise par ce dernier, il est évident que l'enseignant aurait été déclaré coupable. Le juge du procès a d'ailleurs tiré toutes les conclusions nécessaires pour justifier un verdict de culpabilité [...] ([1996] 2 R.C.S. R. c. Audet, c'est nous qui soulignons). Les juges de la Cour suprême infirmeraient avec ce verdict les décisions de non-culpabilité provenant de la Cour d'appel du Nouveau-Brunswick et de la Cour du Banc de la Reine de la même province. Cinq juges de la plus haute cour du Canada ont soutenu - deux juges étaient dissidents ${ }^{1}$-, que du fait de leur situation de confiance, les enseignants doivent prêcher par l'exemple et ils donnent l'exemple autant par leur conduite en dehors des salles de cours que par leur prestation dans celles-ci (cf. Jeffrey, 2013).

Le thème de la confiance est suffisamment important dans l'éthique et le droit des enseignants pour qu'on y porte notre attention. Dans les trois causes citées, les juges considèrent, d'une part, que les enseignants sont présumés en position de confiance face aux élèves, et d'autre part, qu'ils doivent agir pour conserver cette confiance. Dans ce texte bref, nous désirons explorer ce thème de la confiance en lien avec la conception émancipatrice de l'éthique que nous privilégions.

\section{Confiance}

Il n'est pas difficile de montrer, par des exemples, que la confiance joue un rôle fondamental en éthique. Un enfant fait entièrement confiance à ses parents et ces derniers doivent faire complètement confiance aux enseignants. Lorsque dans un milieu de travail les collègues se font confiance, chacun peut, sans hésiter, remettre à n'importe lequel une lettre importante à mettre à la poste. Mais avonsnous le courage moral de remettre une lettre importante à un étranger afin qu'il la mette à la poste? La confiance concerne les sentiments. Nous devons nous sentir en confiance avec autrui pour lui demander un tel service. Mais la confiance relève aussi d'une rationalité particulière qui s'apparente à un calcul de probabilité. Nous nous demandons si nous pouvons faire confiance à tel ou tel individu. Nous calculons, à l'aune de nos expériences personnelles, de la situation, du contexte et du visage de cet individu qui nous est étranger, si nous pouvons lui faire confiance. Il va de soi qu'il est plus facile de faire confiance à un inconnu qui partage notre culture qu'à un individu rencontré dans un pays que nous visitons. Aussi, les normes de sécurité varient d'une société à une autre. Nous savons qu’on ne peut faire confiance à un chauffeur de taxi à New Delhi. Il ne vous amènera pas à votre destination directement. Chacun apprend, par ses propres expériences, mais aussi en entendant les expériences des uns et des autres, qu'on ne peut accorder sa confiance d'une manière égale à toutes personnes.

Les personnes autour de soi, autant les familières que les étrangères, sont considérées comme fiables lorsque nous sommes assurés de leur honnêteté ou lorsque nous pouvons nous abandonner à elles. Lengagement éthique d'une personne garantit la confiance. Lorsqu'il n'est plus possible de faire confiance à un ami ou à un amoureux, alors la relation éthique est mise en péril. Par ailleurs, la vie 
morale disparaît dans les sociétés en guerre puisqu'il faut bien se méfier du voisin qui a peut-être adhéré au clan ennemi. Si on ne peut faire confiance à son propre père ou à ses propres frères sans les surveiller de près, alors la familiarité, la complicité, la convivialité, la solidarité et la sécurité s'avèrent impossibles à partager. Nous ne pouvons devenir des personnes éthiques que dans des liens de confiance (Watier, 2008). Nul enfant ne serait capable de vie morale s'il nétait éduqué dans un milieu social qui suppose des liens de confiance.

\section{Les éthiques et les libertés}

Même si les éthiques ${ }^{2}$ sont plurielles, toutes s'intéressent aux régulations des libertés individuelles (Canto-Sperber, 2001). Dans les sociétés démocratiques comme la nôtre, nous jouissons de plusieurs libertés que ne connaissaient pas les générations antérieures. Pensons notamment à la liberté acquise par les femmes d'accéder à tous les programmes universitaires. Nos chartes, qui nont pas encore un demi-siècle d'existence, ratifient des libertés individuelles qui touchent notamment aux convictions religieuses, à l'expression des idées et à l'orientation sexuelle. Nous possédons des libertés qui font l'envie d'individus qui habitent dans des pays qui tolèrent le racisme, le sexisme et l'homophobie. Mais ces libertés chèrement acquises ont des assises fragiles. Elles doivent être protégées par des institutions démocratiques. En même temps, chaque individu doit apprendre à les connaître, à les utiliser avec sagesse et à les assumer. Une liberté ne pourrait conférer un pouvoir absolu à un individu. Par exemple, on ne pourrait user de sa liberté d'expression sans considérer autrui. Nous jouissons tous des mêmes libertés. Nos libertés commencent là où celles des autres commencent, et s'arrêtent là où celles des autres s'arrêtent. Nous sommes tous libres en même temps. Nous sommes tous égaux en dignité. C'est pourquoi nous devons apprendre à partager nos libertés avec autrui. C'est en ce sens que l'éthique concerne les régulations des libertés individuelles. Si les libertés n'étaient pas régulées, les plus forts pourraient abuser des plus faibles.

Les conceptions de l'éthique se singularisent habituellement par leur position sur : 1 . La reconnaissance des libertés individuelles; 2. Léducation des individus au sujet de ces libertés et 3 . Leurs limitations. Une éthique propose des maximes, des valeurs, des normes, des devoirs, des règles qui signalent aux individus que leurs libertés ne sauraient être sans limites. À côté de l'éthique, les libertés individuelles sont limitées par diverses autres règles issues des mœurs, des us et coutumes, du droit, des pouvoirs politiques et de la religion. Or, les règles de l'éthique se distinguent des autres du fait qu'elles s'établissent sur la reconnaissance de l'autonomie morale ${ }^{3}$ des individus et sur la confiance qu'on leur accorde. Cette confiance s'appuie sur une gageure ou une présomption que chacun a appris à bien se conduire ${ }^{4}$. À cet égard, nous présumons que nous pouvons leur faire confiance. Par exemple, nous présumons que la personne que nous rencontrons dans la rue ou sur un site web ne nous veut pas de mal. Il y a là une gageure, car nous ne pouvons jamais en avoir la totale certitude. Mais nous préférons y croire, sinon la vie en société serait intenable. Nous préférons d'emblée faire confiance aux individus. Lorsqu'une personne que vous fréquentez depuis quelques semaines vous déclare son amour, vous prenez le risque de la croire. Nous présumons que le serveur au restaurant ne va pas abuser de notre confiance en nous trompant sur le coût du repas. Nous présumons que le chauffeur de taxi ne prend pas de détours pour augmenter son salaire. Bien évidemment, le niveau de confiance pourra varier selon les situations, les contextes et les personnes en présence. Mais nous devons reconnaitre que la confiance mutuelle 
est préalable aux relations éthiques émancipatrices. Lorsque les liens de confiance sont brisés, alors l'éthique tend à devenir contraignante, et même oppressante. La méfiance s'installe, les positions se contractent, les communications deviennent plus acerbes, chacun se surveille.

Les étudiants, en début d'année scolaire, présument qu'ils peuvent faire confiance à leurs enseignants. Les enseignants présument que leurs étudiants se conduiront selon les normes de l'éthique universitaire. Lorsque la confiance règne, les régulations éthiques émancipatrices s'imposent d'elles-mêmes. Les étudiants s'attendent à ce que les enseignants agissent avec bienveillance et équité, et les enseignants s'attendent à ce que les étudiants ne trichent pas, ne mentent pas et ne les trompent pas. Lorsqu'on ne se fait plus du tout confiance, les régulations éthiques ne suffisent plus. Il faut alors faire appel aux régulations politiques et juridiques.

\section{Éthique contraignante et éthique émancipatrice}

Les règles de l'éthique se sont constituées historiquement autour de deux grandes orientations: 1. Celles qui encadrent le «savoir-vivre-ensemble» (civisme, civilité, politesse, sécurité, etc.) dans des institutions justes et 2. Celles qui encadrent les conduites individuelles. La première orientation touche aux questions de justice, alors que la seconde concerne la vie bonne (Ricœur, 1990). Or, le thème de la confiance est commun à ces deux orientations, et peut même servir de critère pour différencier les éthiques contraignantes et les éthiques émancipatrices. Habituellement, les tenants des éthiques contraignantes accordent peu de confiance aux individus, alors que les tenants des éthiques émancipatrices leur accordent une grande confiance. L'éthique humaniste ${ }^{5}$ dans laquelle nous nous inscrivons se veut émancipatrice. Elle est fondée sur l'égale valeur des êtres humains en dignité et sur la confiance mutuelle. C'est une éthique qui reconnait aux individus beaucoup de libertés et de responsabilités. Elle est aux antipodes des éthiques contraignantes qui sont arrimées sur des logiques de domination des individus. Ces dernières cherchent à limiter leurs libertés, à contrôler leur agir et même à les asservir. Elles se justifient en s'appuyant sur la difficulté, sinon l'impossibilité de faire confiance aux individus. Par conséquent, ces éthiques se servent de la méfiance pour légitimer l'assujettissement, l'asservissement et l'oppression.

\section{La liberté de conscience et autonomie morale}

Soulignons enfin, pour préciser notre conception émancipatrice de l'éthique, que la liberté de conscience (pouvoir penser et agir autrement) est la condition cognitive de la vie morale. L'activité morale suppose une liberté de conscience qui, peu à peu, affirme et défend ses valeurs et ses convictions d'une manière responsable. La moralité, c'est-à-dire la capacité d'agir moralement, consiste à établir un choix, une décision ou une action sur un critère moral et à en assumer la responsabilité. L'autonomie morale, pour le dire brièvement, renvoie à la capacité de l'individu de trouver des ressources en lui-même pour agir avec dignité et responsabilité. L'individu moralement autonome n'attend pas qu'on lui dise ce qu'il peut faire de bien, il en prend l'initiative.

En termes de développement cognitif, l'acquisition de la liberté de conscience, de l'autonomie morale et de la capacité de responsabilité sont préalables à la reconnaissance des libertés individuelles (expression, pensée, conviction, association, déplacement, travail, etc.). On ne pourrait reconnaître à

Formation et profession 23(3), 2015 
des enfants des libertés individuelles pour lesquelles ils ne pourraient être responsables. La liberté de conscience est un acquis dans l'évolution de l'homo sapiens, mais son apprentissage et son usage sont des phénomènes culturels (Ogien, 2011). En effet, nous ne naissons pas avec une claire connaissance de notre pouvoir d'agir librement et de nos responsabilités. Cette connaissance nous est transmise au cours de notre éducation et chacun de nous en expérimente le cadre et les limites. Les enfants doivent donc être socialisés et éduqués aux libertés individuelles reconnues par leur société. Cette possibilité de se voir confier des libertés individuelles entraîne de facto des responsabilités. Dans toutes les sociétés humaines, les enfants deviennent complètement responsables de leurs actes lorsqu'ils atteignent l'âge adulte ou lorsqu'ils passent avec succès, selon les rites traditionnels, des épreuves initiatiques. Avant l'âge de la maturité, on leur reconnaît peu de libertés individuelles puisqu'ils ne sont pas encore aptes à les assumer. En fait, on les déclare autonomes moralement lorsqu'ils sont réputés capables d'assumer les libertés reconnues aux individus de leur société.

\section{Conclusion}

En somme, nous inscrivant dans une tradition humaniste, notre éthique émancipatrice présuppose, au point de vue sociopolitique, la confiance mutuelle, et au point de vue cognitif, la liberté de conscience et l'autonomie morale de chaque individu. Une telle éthique, plutôt que de contraindre, invite chacun à se conduire avec dignité et d'une manière responsable dans la conduite de sa vie. En effet, une éthique qui vise l'émancipation ne supporte pas les contraintes moralisatrices. Elle ne pourrait utiliser la peur, le chantage et la menace. Elle ne peut que lancer des invitations à tout un chacun afin qu'ils se conduisent de la meilleure façon possible compte tenu des contextes. Dans le monde scolaire, cette invitation prend le sens de mettre de l'avant des valeurs de respect, de bienveillance, d'équité, de justice, de réserve, de responsabilité, et bien d'autres, qui vont guider et orienter le travail des enseignants.

Les enseignants, sensibles à cette éthique, sont invités - et non pas contraints - à se conduire de manière à conserver la confiance des étudiants, des parents, des collègues, des membres de la direction et du public. Ils montrent qu'ils sont dignes de confiance lorsqu'ils agissent, en toutes situations éducatives, avec compétence. Cela signifie qu'ils peuvent expliquer leurs décisions et leurs actions à l'aune des savoirs établis de leur profession. Voilà pourquoi on peut leur faire confiance. 


\section{Notes}

1 L'argument de leur dissidence est le suivant : la Cour aurait dû prouver le lien de confiance et d'autorité étant donné que les élèves n'entretiennent pas univoquement le même type de lien avec leurs enseignants.

2 Nous ne distinguons pas ici l'éthique et la morale puisque ces deux concepts sont passablement synonymes dans l'histoire de la philosophie. En fait, on associe souvent la morale à des contraintes extérieures à l'individu et l'éthique à la conduite personnelle de sa vie. Mais il s'avère que les nouvelles éthiques (environnement, affaires, journalisme, recherches, etc.) proposent autant de contraintes que les vieilles morales religieuses.

3 Au Québec, l'âge de la pleine autonomie morale est 18 ans. Avant cet âge, un jeune n'est pas reconnu entièrement responsable de ses actes. On considère qu'il est encore en apprentissage. C'est pourquoi il n'est pas jugé et sanctionné comme un adulte.

4 Les régulations politiques et juridiques s'appuient également sur l'autonomie morale, mais minimisent la présomption à bien se conduire. C'est pourquoi elles utilisent la répression (radar routier, amendes et contraventions, emprisonnement, etc.). Il n'y a pas de sanction directe en éthique. Si une personne ment et qu'elle est la seule à le savoir, elle pourra uniquement se sentir coupable, infidèle ou traitre. Lorsqu'une personne découvre qu'on lui a menti, elle pourra être fâchée, déçue et frustrée. Elle pourra renier le menteur. Mais il n'y a pas d'autres sanctions, à moins de croire aux sanctions métaphysiques du ciel

5 Nous référons à un humanisme libéral laïque qui affirme son indépendance à l'égard des injonctions religieuses, des doctrines issues de la révélation et des croyances surnaturelles.

\section{Références}

Canto-Sperber, M. (2001). L’inquiétude morale et la vie humaine. Paris : Presses universitaires de France.

Jeffrey, D. (2013). Profession enseignante : de la moralité exemplaire à l'éthique professionnelle. Formation et profession, 21(3), 18-29. Repéré à http://www.formation-profession.net/files/numeros/6/v21_n03 189.pdf

Ogien, R. (2011). L'influence de l'odeur des croissants chauds sur la bonté bumaine. Paris : Grasset.

Watier, P. (2008). Éloge de la confiance. Paris : Belin.

\section{Pour citer cet article}

Jeffrey, D. (2015). Éthique enseignante et confiance. Formation et profession, 23(3), 123-128. http://dx.doi.org/10.18162/fp.2015.a68 